\title{
LEUKOCYTE ACTIVATION IN PRETERM NEWBORN INFANTS OF PREECLAMPTIC MOTHERS
}

\author{
R.C. Silveira, F.R.S. Faulhaber, A.P. Vargas, R.S. Procianoy \\ Universidade Federal do Rio Grande do Sul/Hospital de Clinicas de Porto Alegre, Porto Alegre, Brazil
}

Background: There is scarce information on leukocyte activation in preterm newborns of preeclamptic mothers.

Objective: To study plasma IL-8 and GRO- $\alpha$ levels in preterm newborn infants of preeclamptic.

Design/methods: Newborn infants with gestational age $<36$ weeks and birth weight $<2000$ grams were included in the study, and divided in two groups: non-preeclamptic and preeclamptic groups. Exclusion criteria were: congenital malformations, and congenital infections. When blood sample was collected with laboratory exams in the first 48 hours of life, a small amount was used for IL- 8 and GRO- $\alpha$ measurement. Method utilized was enzyme immunoassay.

Results: 119 preterm infants (64 non-preeclamptic and 55 preeclamptic) were included in the study. Both groups were similar in birth weight, gestational age, Apgar scores at 5 minutes, sepsis, mechanical ventilation, TPN, NEC, IVH and death. The non-preeclamptic group had more rupture of membranes for $>$ 18 hours, vaginal delivery, maternal urinary tract infection and chorioamnionitis. The preeclamptic group had more RDS, neutropenia, low platelet count and SGA. IL-8 was higher in the non-preeclamptic group [259.6 pg/mL (86.4-261.3) and $81.9 \mathrm{pg} / \mathrm{mL}(3.6-87.2) \mathrm{p}<0.001$ in non-preeclamptic and preeclamptic groups, respectively]. GRO- $\alpha$ was similar in both groups [273.9 pg/mL(63.9-306.7) and $261 \mathrm{pg} / \mathrm{mL}$ (116.6321.3) $\mathrm{p}=0.236$ in non-preeclamptic and preeclamptic groups, respectively]. After multiple regression analysis only absence of preeclampsia was associated with high IL-8 levels.

Conclusions: Preterm newborn infants of preeclamptic mothers have a decreased plasma level of IL-8. Our findings suggest that the leukocyte activation may be impaired in infants of preeclamptic mothers. 